\title{
Proteína C Reactiva en la EPOC y su relación con la gravedad de la enfermedad, las exacerbaciones y las comorbilidades
}

\author{
ORLANDO DÍAZ ${ }^{1,3}$, ALEJANDRA PARADA², CRISTÓBAL RAMOS ${ }^{4}$, \\ JULIETA KLAASSEN ${ }^{2}$, JUAN CARLOS DÍAZ ${ }^{4}$, MAX ANDRESEN ${ }^{3}$, \\ CARMEN LISBOA ${ }^{1}$, FERNANDO SALDÍAS ${ }^{1}$
}

\section{C-Reactive protein levels in patients with chronic obstructive pulmonary disease}

Background: Patients with chronic obstructive pulmonary disease (COPD) have elevated serum levels of ultrasensitive $C$ reactive protein (CRPus). This raise may be related directly to COPD and its associated systemic inflammation or secondary to other factors such as smoking status, disease severity, acute exacerbations, or associated complications. Aim: To evaluate the potential causes of raised levels of CRPus in stable COPD patients. Patients and Methods: Cohorts of 133 mild-to-very severe COPD patients (41 current smokers), 31 never-smokers, and 33 current smoker controls were compared. Clinical assessments included body mass index (BMI), fat (FM) and fat-free mass (FFM) measurement by DEXA, forced expiratory volume in one second $\left(F E V_{1}\right)$, arterial oxygen tension $\left(\mathrm{PaO}_{2}\right)$, six-minute walking test (SMWT), emphysema (EMPH) and right thigh muscle cross-sectional area (TMCSA), both quantified by high resolution computed tomography. Results: Serum CRPus levels were significantly higher in COPD patients than in controls $(7 \pm 4.2$ and $3.7 \pm 2.7$ $m g / L$ respectively; $p<0.0001)$. Being smoker did not influence CRPus levels. These levels were significantly correlated with FM ( $r=0.30), B M I(r=0.21), F E V_{1}$ $(r=-0.21)$, number of acute exacerbations of the disease in the last year $(r=0.28)$, and $\mathrm{PaO}_{2}(r=-0.27)$. Using multivariate analysis $\mathrm{FM}, \mathrm{PaO}_{2}$, and number of acute exacerbations of the disease in the last year had the strongest association with CRPus levels. Conclusions: CRPus is elevated in COPD patients, independent of smoking status. It is weakly associated with fat mass, arterial oxygen tension and frequency of exacerbations.

(Rev Med Chile 2012; 140: 569-578).

Key words: Emphysema; C-reactive protein; Pulmonary disease, chronic obstructive; Systemic inflammatory response syndrome.

\section{L} a enfermedad pulmonar obstructiva crónica (EPOC) se define actualmente como una entidad prevenible y tratable, caracterizada por limitación del flujo aéreo que no es completamente reversible, y con efectos extrapulmonares que pueden contribuir a su gravedad ${ }^{1}$. Se ha observado además que se asocia con frecuencia a otras comorbilidades y a la elevación de marcadores de inflamación sistémica en la sangre periférica ${ }^{2,3}$.

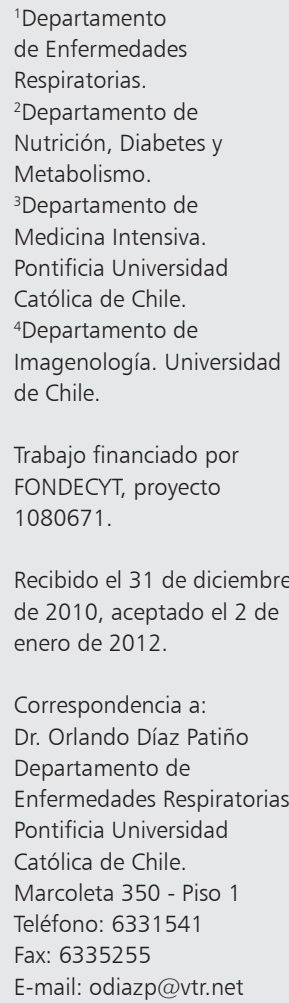

Se ha planteado que podría existir un nexo entre la inflamación sistémica, la gravedad de la EPOC, sus efectos extrapulmonares y las comorbilidades ${ }^{3,4}$, de tal forma que tratando la inflamación sistémica eventualmente se podría modificar la historia natural de la enfermedad ${ }^{2,3}$. Esto se debe a que la inflamación se asocia a una pérdida acelerada de la función pulmonar y a mayor mortalidad ${ }^{5,6}$, y porque tanto los efectos extrapulmonares como 
las comorbilidades pueden contribuir significativamente a dicha mortalidad ${ }^{7,8}$. Se han utilizado diferentes marcadores de inflamación, siendo la proteína $\mathrm{C}$ reactiva ultrasensible (PCRus) sérica uno de los más comúnmente medidos ${ }^{3}$, ya que se considera capaz de reflejar la carga inflamatoria sistémica total'.

De acuerdo a estos estudios, la PCRus se ha encontrado elevada en pacientes con EPOC estable en comparación con controles ${ }^{3}$, siendo sus niveles séricos mayores en presencia de tabaquismo activo ${ }^{10}$. Asimismo, los valores aumentarían con la gravedad de la enfermedad, evaluada con índices funcionales como el $\mathrm{VEF}^{10}{ }^{10}$, la magnitud de la hiperinflación pulmonar ${ }^{11}$, la presencia de hipoxemia ${ }^{11}$, o con índices clínicos, como la frecuencia anual de exacerbaciones ${ }^{12}$. También se ha observado que los valores de PCRus son más altos cuando existe compromiso extrapulmonar, ya sea por pérdida de la masa muscular periférica o por reducción de la capacidad de ejercicio ${ }^{13}$. Otros índices que reflejan la gravedad de la enfermedad, como la magnitud del enfisema, el compromiso de la masa muscular de extremidades inferiores y las alteraciones de la composición corporal, han sido escasamente evaluados ${ }^{14,15}$. Por otro lado, los niveles de PCRus son mayores en los pacientes con EPOC que presentan comorbilidades asociadas, como la enfermedad cardiovascular aterosclerótica $(\mathrm{ECA})^{16}$; caquexia ${ }^{17}$, obesidad ${ }^{18}$; y cáncer pulmonar ${ }^{19}$, entre otras ${ }^{20,21}$. Sin embargo, los estudios que han intentado buscar una asociación entre estos factores y la inflamación, estimando la contribución individual de la gravedad de la EPOC, sus efectos extrapulmonares y las comorbilidades asociadas, sobre los valores séricos de PCRus son escasos y han encontrado hasta ahora asociaciones débiles ${ }^{11,15,22,23}$. Estos resultados negativos podrían explicarse en parte porque los índices de gravedad incluidos han sido exclusivamente espirométricos, mientras que los efectos extrapulmonares han sido pobremente evaluados.

En consecuencia, los objetivos de este estudio han sido establecer si la condición de fumador activo; la gravedad de la EPOC, evaluada por índices espirométricos y medición directa del enfisema; la frecuencia de las exacerbaciones; las manifestaciones extrapulmonares de la enfermedad, evaluadas con estudios de composición corporal y estimación de la masa muscular esquelética; y ciertas comorbilidades frecuentes influyen inde- pendientemente sobre los valores de la PCRus, empleada en este estudio como marcador del grado de inflamación sistémica en la EPOC.

\section{Pacientes y Método}

\section{Pacientes}

Se incorporaron 133 pacientes portadores de EPOC leve a muy grave, según la definición de GOLD (Global Initiative for Obstructive Chronic Lung Disease $)^{1}$, en condiciones estables de su enfermedad, es decir, sin exacerbaciones en los dos meses previos. Estos pacientes fueron reclutados desde la consulta externa, del laboratorio de función pulmonar y del hospital clínico de nuestra institución. Forman parte de una cohorte de seguimiento cuyos criterios de ingreso ya han sido previamente publicados ${ }^{24}$ y a quienes los investigadores responsables del estudio controlan una vez al año, sin participar de su tratamiento.

\section{Controles}

Se reclutaron 64 controles, fumadores y nunca fumadores, que también forman parte de esta cohorte. Este grupo está conformado por voluntarios de nuestra institución, sujetos que consultaron en el policlínico en quienes se descartó enfermedad pulmonar, y por vecinos de los pacientes, invitados a participar por vía telefónica. Los criterios de reclutamiento fueron: a) edad $\geq 45$ años; $\mathrm{VEF}_{1}>80 \%$ y $\mathrm{VEF}_{1} / \mathrm{CVF} \geq 70 \%$ después de recibir $200 \mu \mathrm{g}$ de salbutamol; $\mathrm{yb}$ ) ausencia de antecedentes de asma u otra enfermedad pulmonar crónica.

En los pacientes y controles se excluyó a aquellos participantes que padecieran comorbilidades que impidieran efectuar una prueba de ejercicio, específicamente enfermedades del aparato locomotor o secuelas de accidentes cerebro-vasculares; o condiciones caracterizadas por inflamación sistémica, como las enfermedades neoplásicas activas, las infecciones crónicas y las enfermedades colágeno-vasculares. Asimismo, ningún participante presentaba infecciones intercurrentes al momento de efectuar las mediciones.

Los pacientes y los controles aceptaron participar en el protocolo firmando un acta de consentimiento informado aprobada por el Comité de Ética de la Pontificia Universidad Católica de Chile. 


\section{Mediciones}

En todos los participantes las mediciones se efectuaron en un lapso no mayor a una semana. Se midió peso y talla, y se calculó el índice de masa corporal (IMC). La magnitud de la disnea se evaluó con la escala modificada del Medical Research Council (MMRC) ${ }^{25}$. La historia de tabaquismo incluyó la condición de fumador (nunca, ex fumador y fumador activo) y el consumo de tabaco (paquetes/año). La condición de ex fumador se confirmó con cotinina urinaria, un metabolito de la nicotina, $<10 \mathrm{mg} / \mathrm{L}$. En los pacientes se registró el número de exacerbaciones en los 12 meses previos a ingresar al estudio y el empleo de corticoides inhalados, ya que se ha descrito que su uso puede reducir los niveles séricos de PCRus ${ }^{22}$. Las exacerbaciones fueron definidas como un empeoramiento agudo y sostenido de la condición del paciente, que requirió un cambio en la terapia ${ }^{26}$. Entre las comorbilidades se investigó la presencia o antecedente de hipertensión arterial, hipercolesterolemia y diabetes mellitus y se consignó el antecedente de cardiopatía coronaria, enfermedad vascular periférica y accidente vascular cerebral. Estas comorbilidades se analizaron por separado y también fueron categorizadas según los criterios utilizados por de Torres y cols ${ }^{11}$, como ECA conocida (cardiopatía coronaria, enfermedad vascular periférica o accidente vascular cerebral) o riesgo de ECA (hipertensión arterial, hipercolesterolemia y diabetes mellitus) ${ }^{27}$. El estado nutricional se categorizó empleando el IMC de acuerdo a los criterios de la Organización Mundial de la Salud, pero para los análisis también se utilizó la variable continua, ya que en un extremo $\left(<18,5 \mathrm{~kg} / \mathrm{m}^{2}\right.$; bajo peso) este índice expresa la gravedad de la EPOC, mientras que en el otro extremo $(\geq 30 \mathrm{~kg} /$ $\mathrm{m}^{2}$; obesidad) constituye una comorbilidad.

A todos los participantes se les efectuó una espirometría ${ }^{28}$ y se midió la capacidad inspiratoria (CI), expresando sus resultados como porcentaje de los valores teóricos ${ }^{29,30}$. Además, se midió gases arteriales y la distancia recorrida (DR6) en la prueba de caminata de 6 minutos $^{31}$. La composición corporal se evaluó empleando absorciometría de rayos X duales (DEXA), obteniéndose los valores de la masa libre de grasa (MLG) y la masa grasa (MG). Para la cuantificación del enfisema se realizó una tomografía computada de tórax con un equipo multidetector de 64 canales (Somatom Sensation 64; Siemens Healthcare, Erlangen,
Alemania). El enfisema se definió como las áreas de baja atenuación (ABA) inferiores a -960 Unidades Hounsfield $(\mathrm{UH})^{32}$, y se expresa como el porcentaje de ABA para el volumen pulmonar total (\%ABA-960). En la misma sesión se obtuvo el área de sección transversal de la musculatura del muslo derecho (ASTM), de acuerdo a la técnica descrita por Bernard y cols ${ }^{33}$. La PCRus se obtuvo mediante inmunoensayo enzimático (BioMedica Inc., Newport Beach, California, Estados Unidos de Norteamérica), cuya sensibilidad analítica es de $0,1 \mathrm{mg} / \mathrm{L}$.

\section{Análisis estadístico}

Las variables se expresan como promedios y desviaciones estándar o como números y porcentajes. Debido a su distribución asimétrica, la PCRus se transformó a su logaritmo natural para los análisis (log PCRus). Para explorar posibles diferencias del log PCRus entre pacientes y controles empleamos regresión logística utilizando ambos grupos como variable dependiente binaria y log PCRus como variable independiente, ajustando por potenciales confundentes que pueden influir en los niveles de PCRus: edad; género; historia de tabaquismo (paquetes/año); condición de fumador (nunca fumadores, ex fumadores y fumadores activos); IMC; y comorbilidades. Dichos resultados se expresan como odds ratio (OR) e intervalo de confianza de 95\% (IC 95\%). En los pacientes, las posibles diferencias de los valores del log PCRus según género, uso de corticoides inhalados y presencia de comorbilidades se evaluaron con modelos lineales generales usando el IC 95\% para la estimación de las diferencias y ajustando por índices conocidos de gravedad de la EPOC. Las correlaciones entre log PCRus y las diferentes variables analizadas se estimaron usando correlaciones de Pearson o Spearman, dependiendo de la naturaleza de la segunda variable. Por último, para definir las variables con mayor asociación al log PCRus se empleó regresión lineal múltiple paso a paso. En todos los análisis, se consideró significativo un valor de $\mathrm{p} \leq 0,05$.

\section{Resultados}

Los 133 pacientes representaban las cuatro categorías GOLD: etapa 1, $\mathrm{n}=29$ (22\%); etapa 2, $\mathrm{n}=51(38 \%)$; etapa $3, \mathrm{n}=31(23 \%)$; etapa $4, \mathrm{n}=22$ 
(17\%). De ellos, 41 (31\%) eran fumadores activos, $84(63 \%)$ empleaba corticoides inhalados en dosis variables (budesonida: 200-800 $\mu \mathrm{g} /$ día; fluticasona: $250-1000 \mu \mathrm{g} /$ día), ninguno usaba corticoides sistémicos y $23(17 \%)$ recibían estatinas. Ochenta pacientes presentaron entre 1 y 10 exacerbaciones durante el año previo al estudio, mientras que 53 no tuvieron exacerbaciones. En los 64 controles, 31 (48\%) nunca había fumado y el resto era fumador activo. Once controles (17\%) utilizaban estatinas.

Las características de los cuatro subgrupos categorizados según la condición de fumador se detallan en la Tabla 1. Hubo una diferencia significativa en la distribución por sexo $(\mathrm{p}=0,043)$ debido al predominio de las mujeres entre los controles nunca fumadores y de los hombres en los pacientes ex fumadores. No se observaron diferencias en el IMC, la MG, la MLG ni en la frecuencia de comorbilidades entre los cuatro subgrupos. $\mathrm{Al}$ restringir la comparación a pacientes y controles, la hipertensión arterial fue más frecuente en los pacientes; mientras que en los controles fue más frecuente la dislipidemia y la diabetes mellitus (27 vs $15 \% ; p=0,052)$. La obesidad y la presencia o riesgo de ECA fueron similares en ambos grupos.

Las características funcionales, la magnitud del enfisema y el área de sección transversal del muslo de los cuatro subgrupos se describen en la Tabla 2, donde también se comparan los valores de PCRus. Los pacientes ex fumadores tenían menor relación $\mathrm{VEF}_{1} / \mathrm{CVF}$, mayor porcentaje de enfisema, menor DR6 y mayor disnea que los demás subgrupos. El $\log$ PCRus fue similar entre los sujetos controles nunca fumadores y fumadores y entre los pacientes con EPOC fumadores activos y ex fumadores. En cambio, fue significativamente superior en los dos subgrupos de pacientes en comparación con los dos subgrupos control (Tabla 2; Figura 1). En consecuencia, el $\log$ PCRus fue significativamente mayor en los pacientes con EPOC, siendo el OR ajustado de 3,2 (IC 95\%: 1,7 a 6,3; $\mathrm{p}<0,001$ ).

En los pacientes, las comparaciones de log

Tabla 1. Características demográficas y clínicas de la población en estudio

\begin{tabular}{|c|c|c|c|c|c|c|}
\hline & Controles & $\begin{array}{c}\text { Controles } \\
\text { nunca } \\
\text { fumadores }\end{array}$ & $\begin{array}{l}\text { Controles } \\
\text { fumadores }\end{array}$ & EPOC & $\begin{array}{c}\text { EPOC } \\
\text { fumadores }\end{array}$ & $\begin{array}{c}\text { EPOC } \\
\text { ex } \\
\text { fumadores }\end{array}$ \\
\hline$n$ & 64 & 31 & 33 & 133 & 41 & 92 \\
\hline Sexo, $H / M$ & $27 / 37$ & $12 / 19$ & $15 / 18$ & $79 / 54^{*}$ & $20 / 21$ & $59 / 33$ \\
\hline Edad, años & $63 \pm 9,5$ & $64 \pm 11$ & $62 \pm 8$ & $66 \pm 8,9^{*}$ & $62 \pm 9$ & $68 \pm 9^{b, c}$ \\
\hline $\mathrm{IMC}, \mathrm{kg} / \mathrm{m}^{2}$ & $27 \pm 4$ & $27 \pm 4$ & $27 \pm 4$ & $26,7 \pm 4$ & $26 \pm 4$ & $27 \pm 4$ \\
\hline Historia tabaquismo, paquetes/año & $14 \pm 19$ & $0 \pm 0$ & $27 \pm 18$ & $49 \pm 24^{*}$ & $45 \pm 21^{\mathrm{a}, \mathrm{b}}$ & $51 \pm 25^{a, b}$ \\
\hline Obesidad, n (\%) & $13(20)$ & $6(19)$ & $7(21)$ & $28(21)$ & $8(19,5)$ & $20(21,7)$ \\
\hline Diabetes mellitus, n (\%) & $17(26,6)$ & $10(32)$ & $7(21)$ & $20(15)$ & $4(9,8)$ & $16(17,4)$ \\
\hline Hipercolesterolemia, n (\%) & $34(53)$ & $17(54,8)$ & $17(51,5)$ & $46(34,6)$ & $13(31,7)$ & $33(35,9)$ \\
\hline Hipertensión arterial, n (\%) & $20(31)$ & $11(35,5)$ & $9(27,3)$ & $72(54)$ & $20(48,8)$ & $52(56,5)^{b}$ \\
\hline $\begin{array}{l}\text { Accidente vascular cerebral sin } \\
\text { secuelas, } \mathrm{n}(\%)\end{array}$ & $1(1,6)$ & $0(0)$ & $1(3)$ & $2(1,5)$ & $1(2,4)$ & $1(1,1)$ \\
\hline $\begin{array}{l}\text { Enfermedad vascular periférica, } \\
n(\%)\end{array}$ & $0(0)$ & $0(0)$ & $0(0)$ & $3(2,3)$ & $1(2,4)$ & $2(2,2)$ \\
\hline Cardiopatía coronaria, n (\%) & $2(3,1)$ & $0(0)$ & $2(6,1)$ & $8(6)$ & $0(0)$ & $8(8,7)$ \\
\hline Riesgo ECA, n (\%) ${ }^{\dagger}$ & $42(66)$ & $21(67,8)$ & $21(63,6)$ & $78(58,6)$ & $25(60,9)$ & $53(57,6)$ \\
\hline ECA, n (\%) & $3(4,7)$ & $0(0)$ & $3(9,1)$ & $12(9)$ & $2(4,9)$ & $10(10,9)$ \\
\hline
\end{tabular}

Los valores se expresan como promedios \pm 1 desviación estándar. H: hombres; M: mujeres; IMC: índice de masa corporal; ECA: enfermedad cardiovascular aterosclerótica. ${ }^{\mathrm{a}} \mathrm{p}<0,05$ comparado con control nunca fumador; ${ }^{\mathrm{b}} \mathrm{p}<0,05$ comparado con control fumador; ${ }^{c} p<0,05$ comparado con EPOC fumador. ${ }^{*} p<0,05$ EPOC vs controles. ${ }^{\dagger}$ No se incluye tabaquismo como riesgo, sólo hipertensión arterial, hipercolesterolemia y diabetes mellitus. 
Tabla 2. Función pulmonar, disnea, magnitud del enfisema, área de sección transversal del muslo y capacidad de ejercicio de la población en estudio

\begin{tabular}{|c|c|c|c|c|c|c|}
\hline & Controles & $\begin{array}{c}\text { Controles } \\
\text { nunca } \\
\text { fumadores }\end{array}$ & $\begin{array}{l}\text { Controles } \\
\text { fumadores }\end{array}$ & EPOC & $\begin{array}{c}\text { EPOC } \\
\text { fumadores }\end{array}$ & $\begin{array}{c}\text { EPOC } \\
\text { ex } \\
\text { fumadores }\end{array}$ \\
\hline $\mathrm{VEF}_{1}, \%$ teórico & $108 \pm 18$ & $115 \pm 20$ & $103 \pm 13$ & $60 \pm 24^{*}$ & $66 \pm 24^{a, b}$ & $57 \pm 23^{a, b}$ \\
\hline $\mathrm{VEF}_{1} / \mathrm{CVF}, \%$ & $77 \pm 4$ & $78 \pm 4$ & $75 \pm 4$ & $46 \pm 14^{*}$ & $51 \pm 14^{a, b}$ & $44 \pm 14^{\mathrm{a}, \mathrm{b}, \mathrm{c}}$ \\
\hline $\mathrm{Cl}$ \% teórico & $101 \pm 18$ & $101 \pm 19$ & $100 \pm 18$ & $82 \pm 22 *$ & $84 \pm 20^{a, b}$ & $81 \pm 23^{a, b}$ \\
\hline $\mathrm{PaO}_{2}, \mathrm{mmHg}$ & $81 \pm 8$ & - & $81 \pm 8$ & $73 \pm 10^{*}$ & $74 \pm 11^{b}$ & $72 \pm 10^{b}$ \\
\hline Disnea MMRC, puntos & $0 \pm 0$ & $0 \pm 0$ & $0 \pm 0$ & $1,6 \pm 1,1^{*}$ & $1,3 \pm 1,1^{a, b}$ & $1,8 \pm 1,1^{a, b, c}$ \\
\hline \%ABA-960 UH, \% & $5,1 \pm 3,8$ & $4,7 \pm 3,8$ & $5,4 \pm 3,8$ & $16 \pm 10^{*}$ & $10,7 \pm 8^{a}$ & $19 \pm 10^{a, b, c}$ \\
\hline ASTM, $\mathrm{cm}^{2}$ & $68 \pm 22$ & $66 \pm 23,5$ & $69 \pm 20$ & $62 \pm 20$ & $65 \pm 23$ & $61 \pm 18$ \\
\hline MG, kilos & $26 \pm 7,8$ & $25 \pm 7,2$ & $27 \pm 8,0$ & $25,9 \pm 9,1$ & $25 \pm 8,5$ & $26 \pm 9,4$ \\
\hline MLG, kilos & $41 \pm 9,7$ & $40 \pm 9,4$ & $42 \pm 9,9$ & $42 \pm 8,6$ & $42 \pm 9,3$ & $42 \pm 8,3$ \\
\hline DR6, metros & $574 \pm 75$ & $572 \pm 83$ & $577 \pm 69$ & $472 \pm 106^{*}$ & $506 \pm 106^{a, b}$ & $457 \pm 102^{a, b, c}$ \\
\hline Exacerbaciones, $\mathrm{n}$ & - & - & - & $1,56 \pm 2^{*}$ & $1,54 \pm 2,4$ & $1,57 \pm 1,8$ \\
\hline PCRus, mg/L & $3,7 \pm 2,7$ & $3,25 \pm 2,5$ & $4,21 \pm 2,9$ & $7 \pm 4,2^{*}$ & $7,1 \pm 3,4^{a, b}$ & $6,98 \pm 4,5^{\mathrm{a}, \mathrm{b}}$ \\
\hline Log PCRus & $1,0 \pm 0,87$ & $0,86 \pm 0,87$ & $1,15 \pm 0,87$ & $1,7 \pm 0,77^{*}$ & $1,77 \pm 0,72^{a, b}$ & $1,67 \pm 0,80^{a, b}$ \\
\hline
\end{tabular}

Los valores se expresan como promedios \pm 1 desviación estándar. VEF : volumen espiratorio forzado del primer segundo; CVF: capacidad vital forzada; Cl: capacidad inspiratoria; disnea MMRC: escala modificada de disnea del Medical Research Council; \%ABA-960 UH: porcentaje de áreas de baja atenuación menores a -960 Unidades Hounsfield; ASTM: área de sección transversal de la musculatura del muslo derecho; MG: masa grasa; MLG: masa libre de grasa; DR6: distancia recorrida en la prueba de caminata de 6 minutos; PCRus: proteína C reactiva ultrasensible. ${ }^{a} p<0,05$ comparado con control nunca fumador; ${ }^{b} p<0,05$ comparado con control fumador; ${ }^{c} p<0,05$ comparado con EPOC fumador. ${ }^{*} p<0,001$ EPOC vs controles.

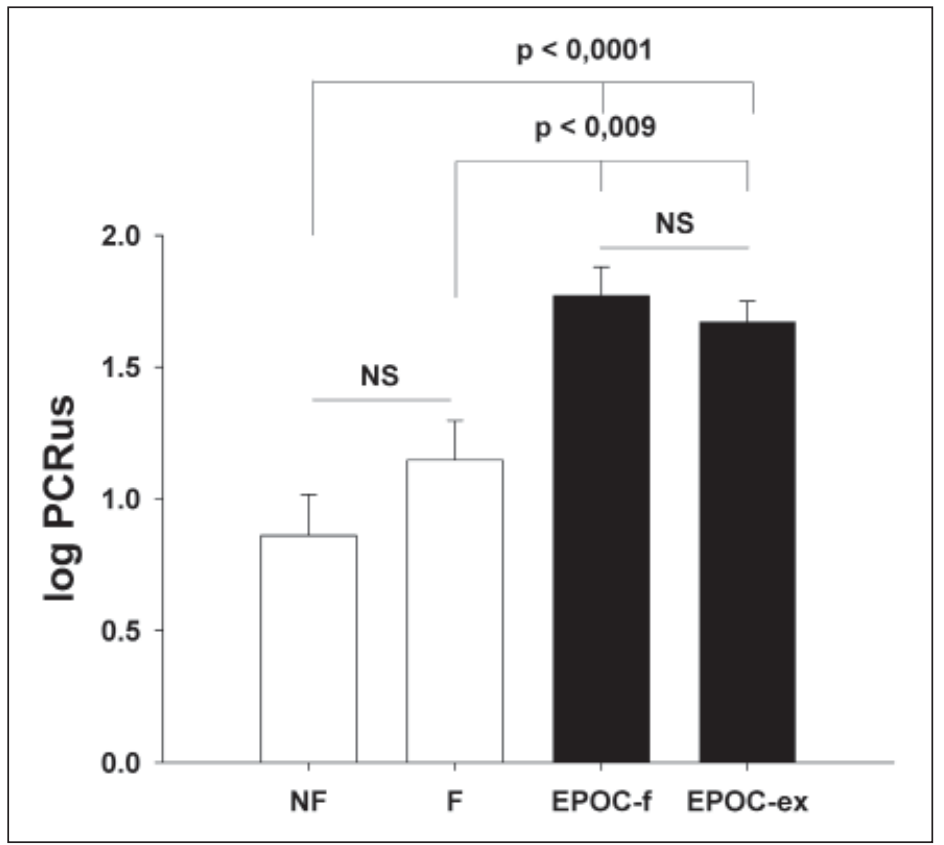

Figura 1. Comparación de los valores del logaritmo natural de la proteína c reactiva ultrasensible entre controles nunca fumadores y fumadores y pacientes con enfermedad pulmonar obstructiva crónica fumadores y ex fumadores. Los valores se expresan como promedios \pm 1 desviación estándar. Los valores de p corresponden a ANOVA de una vía y comparaciones múltiples con corrección de Bonferroni. NF: controles nunca fumadores; F: controles fumadores; EPOC: enfermedad pulmonar obstructiva crónica; EPOC-f: EPOC fumadores activos; EPOC-ex: EPOC ex fumadores; log PCRus: logaritmo natural de proteína c reactiva ultrasensible; NS: no significativo. 


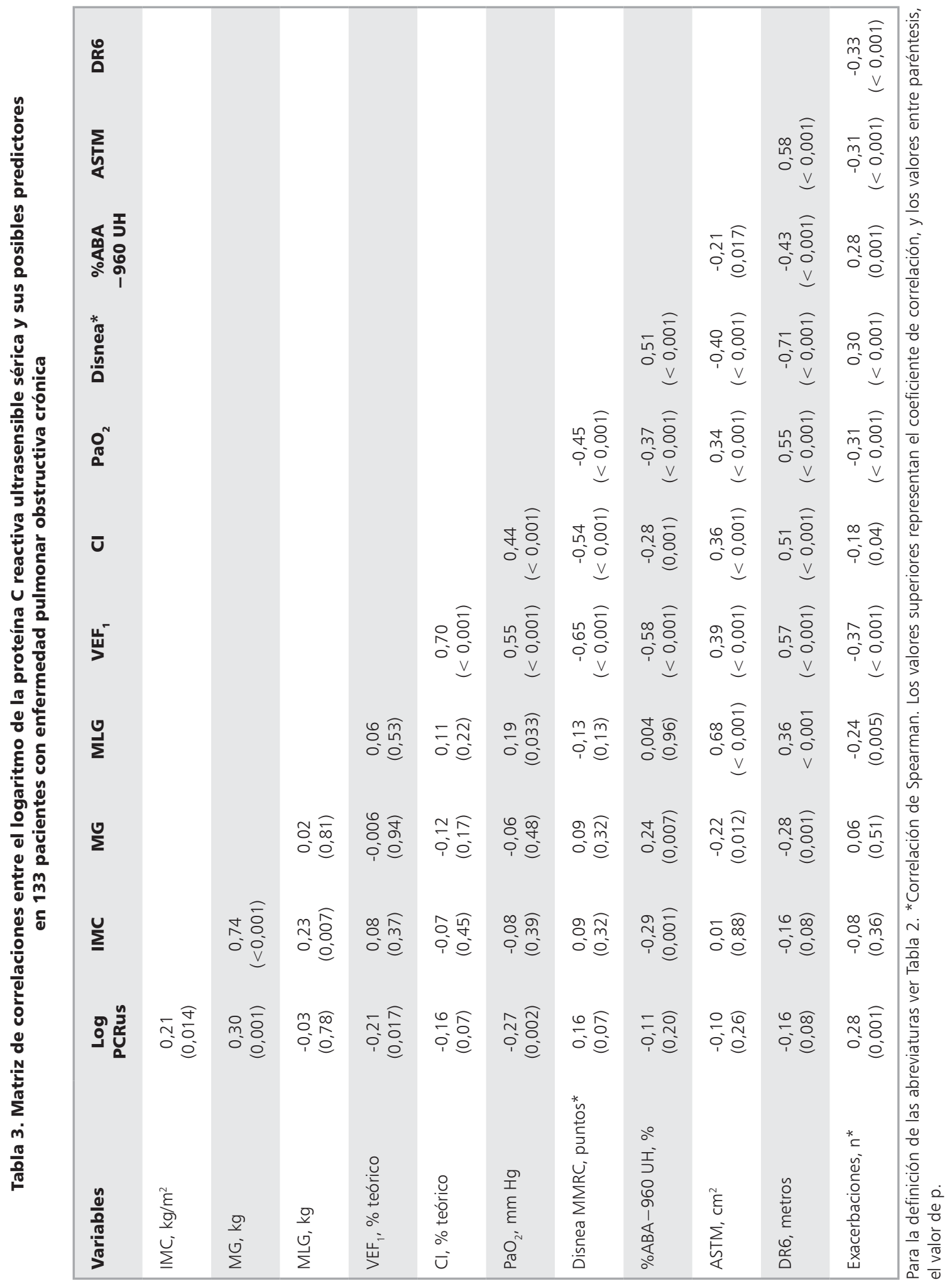



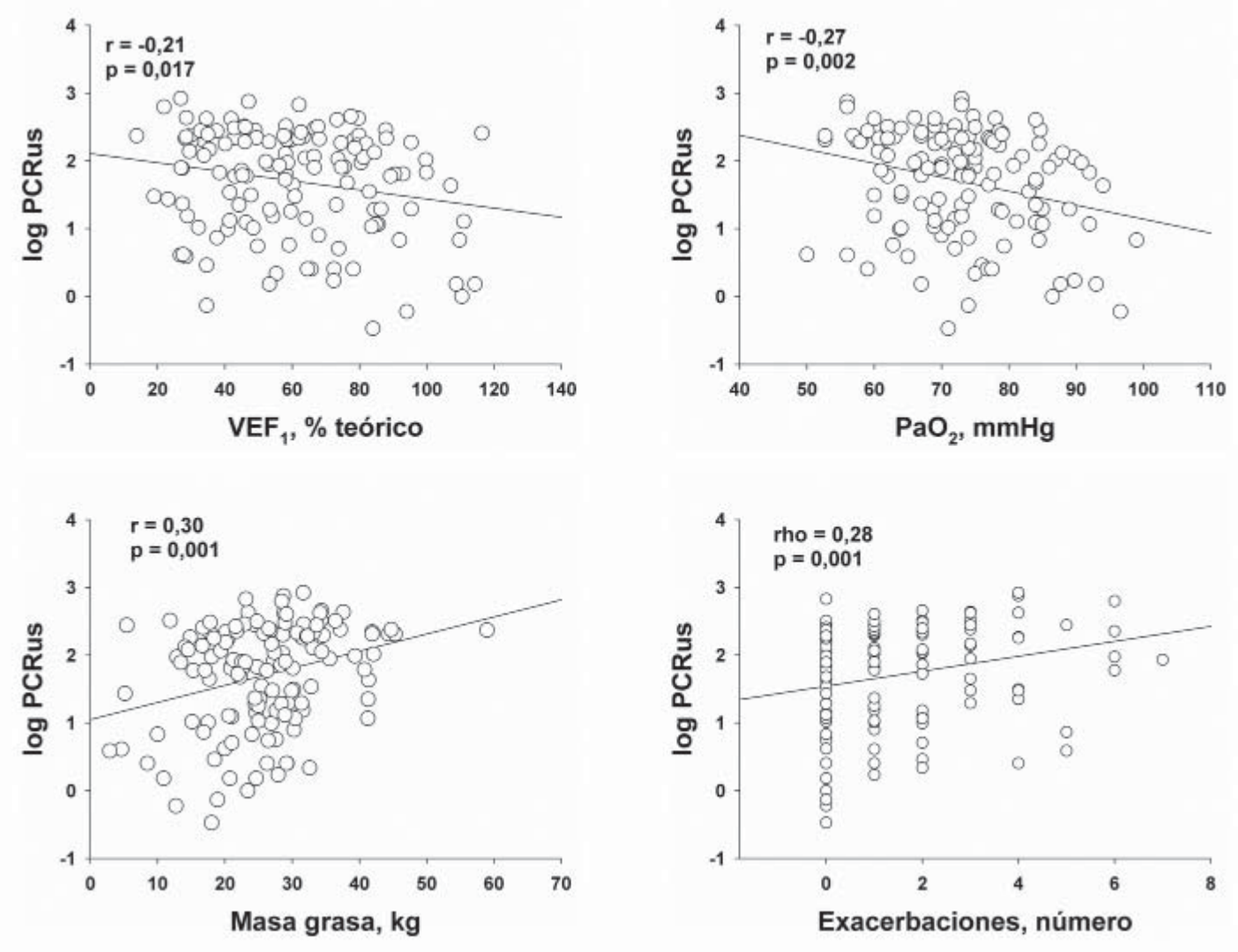

Figura 2. Variables significativamente asociadas con el logaritmo de PCRus en 133 pacientes con enfermedad pulmonar obstructiva crónica.

PCRus ajustadas por VEF, IMC, $\mathrm{PaO}_{2}, \mathrm{DR} 6$ y número de exacerbaciones no mostraron diferencias estadísticas para el género, el uso de corticoides inhalados, la presencia de hipertensión arterial, la diabetes mellitus, la dislipidemia, el antecedente de ECA o riesgo de ECA. En cambio, el log PCRus fue significativamente mayor en obesos (IC 95\% 0,023 a 0,$67 ; p=0,036$ ).

En los pacientes, el log PCRus se correlacionó significativamente con el $\mathrm{VEF}_{1}$, el IMC, la MG, la $\mathrm{PaO}_{2}$ y el número de exacerbaciones del año previo (Tabla 3; Figura 2). No se observó una correlación significativa con la MLG, el \%ABA-960, el ASTM ni la DR6. En el análisis de regresión múltiple, sólo fueron seleccionadas la $\mathrm{PaO}_{2}$, la $\mathrm{MG}$ y el número de exacerbaciones como las variables más estrechamente asociadas al log PCRus. Sin embargo, estas tres variables apenas explicaron el 18\% de la variabilidad del log PCRus.

\section{Discusión}

Nuestros resultados confirman que la PCRus se encuentra elevada en los pacientes con EPOC estable en comparación con sujetos controles de edad semejante. En ambos grupos sus niveles son independientes de la condición de fumador. En los pacientes con EPOC existe una discreta asociación de la PCRus con la gravedad de la enfermedad y con la masa grasa, pero no con la presencia de comorbilidades.

La elevación de la PCRus en la EPOC estable en comparación con sujetos controles ha sido demostrada en numerosos estudios, incluyendo una comunicación de nuestro grupo ${ }^{34}$. En el presente estudio estos datos se han confirmado ajustando por potenciales confundentes, y son compatibles con un estado inflamatorio sistémico propio de la EPOC. 
En los pacientes con EPOC y controles, los valores de la PCRus no se asociaron a la condición de fumador activo. Aunque ello coincide con resultados obtenidos en cohortes pequeñas ${ }^{11,22}$, estudios poblacionales han mostrado un efecto pro-inflamatorio del tabaquismo activo tanto en sujetos normales ${ }^{35}$ como en pacientes con EPOC ${ }^{10}$. Tales discrepancias podrían deberse a la incapacidad de los estudios poblacionales para ajustar por las variables confundentes apropiadas, como las exacerbaciones y las comorbilidades. También puede deberse al menor poder estadístico de las cohortes pequeñas, entre las que se incluye nuestro estudio. En cualquier caso, la ausencia de significación en cohortes pequeñas sugiere que los efectos del tabaquismo activo son probablemente marginales. Así, nuestros datos insinúan que la inflamación es gatillada cuando la EPOC ya está presente y que se mantendría en el tiempo independientemente del acto de fumar. En línea con esta hipótesis, recientemente se ha demostrado que polimorfismos del gen para PCR no predicen el desarrollo subsecuente de EPOC ${ }^{36}$. Así, la elevación de la PCR sérica podría ser influenciada por la enfermedad en sí misma ${ }^{36,37}$ o por factores relacionados a ella, como su gravedad o la presencia de comorbilidades.

Nuestros resultados confirman que factores relacionados con la gravedad de la EPOC, como el $\mathrm{VEF}_{1}$, la $\mathrm{PaO}_{2}$ y las exacerbaciones, y otros relacionados con las comorbilidades, como el IMC y la MG explican parte de la variabilidad de la PCR sérica, si bien marginalmente. Sin embargo, sólo la $\mathrm{PaO}_{2}$, las exacerbaciones y la MG mantuvieron su asociación con la PCRus en el análisis multivariado. La exclusión del $\mathrm{VEF}_{1}$, índice tradicionalmente empleado para expresar la gravedad de la EPOC, podría deberse en parte a su significativa asociación tanto con la $\mathrm{PaO}_{2}$, como con las exacerbaciones (Tabla 3). La correlación negativa entre la $\mathrm{PaO}_{2}$ y la PCRus, destacada previamente por de Torres y $\operatorname{cols}^{11}$, se ha atribuido a que la hipoxemia, incluso intermitente, podría gatillar estrés oxidativo e inflamación ${ }^{38,39}$. Otros índices de la gravedad de la enfermedad, como la hiperinflación pulmonar y la magnitud del enfisema no se correlacionaron con el $\log$ PCRus. Estos hallazgos coinciden, respectivamente, con la débil asociación encontrada por de Torres y cols ${ }^{11}$ para la hiperinflación pulmonar, y por Papaioannou y cols ${ }^{14}$, para el enfisema pulmonar, si bien estos últimos autores estudiaron un grupo menor de pacientes y evaluaron el enfisema sólo visualmente. Ambos índices han sido sugeridos como potenciales promotores de inflamación sistémica ${ }^{40}$, hipótesis no sustentada por nuestros resultados. Por otro lado, la correlación directa entre las exacerbaciones y el log PCRus es consistente con un efecto acumulativo en el tiempo de los marcadores inflamatorios como resultado de dichas exacerbaciones, ya descrito por Donaldson y cols ${ }^{5}$.

Es interesante destacar que la relación directa entre la PCRus y la MG, pero no con la MLG ni con el ASTM, contradice el concepto usualmente aceptado de que la caquexia gatillaría la inflamación en la $\mathrm{EPOC}^{17}$. Sin embargo, confirma otros datos recientes que sugieren que es el tejido adiposo el que contribuye a la inflamación sistémica de la EPOC $^{41,42}$ y coincide con la elevación de la PCR observada en pacientes obesos con EPOC ${ }^{18}$. La ausencia de correlación con la MLG y el ASTM, ambas estrechamente relacionadas entre sí (Tabla 3 ), sugieren que la masa muscular periférica no es afectada por la inflamación, y es consistente además con la débil asociación observada entre la DR6 y el log PCRus, ya que la DR6 depende a su vez de la masa muscular periférica.

Con excepción de la obesidad, las comorbilidades no influyeron en los valores de log PCRus. Es particularmente notoria la ausencia de asociación entre el log PCRus, las comorbilidades cardiovasculares y sus factores de riesgo, que podría explicarse por varias razones: a) sesgo de selección, ya que sólo se incluyeron pacientes capaces de tolerar una prueba de caminata de 6 minutos, excluyendo a aquellos más graves; b) influencia de tratamientos previos (estatinas y corticoides inhalados), que pueden haber reducido la PCRus en el grupo de mayor riesgo ${ }^{43}$; y c) limitaciones propias de emplear criterios de riesgo, cuyo rendimiento es variable ${ }^{43,44}$, en lugar de eventos cardiovasculares, que requieren estudios longitudinales. Sin embargo, también es posible que la ausencia de asociación se deba a que ésta es relativamente débil, como ha sido demostrado recientemente ${ }^{45}$. Los resultados disímiles encontrados en pacientes con EPOC podrían explicarse por esta razón y por los diferentes métodos empleados para definir el riesgo cardiovascular ${ }^{11,16,22,46}$.

El presente estudio posee algunas limitaciones adicionales a las ya mencionadas en el párrafo anterior. Por un lado, se han empleado criterios 
de reclutamiento que excluyen a los individuos más graves, por lo que sus resultados no pueden generalizarse a toda la población de pacientes con EPOC. Además, al ser un estudio transversal, las asociaciones encontradas no necesariamente reflejan causalidad.

En resumen, en la EPOC estable existiría un estado de inflamación sistémica en que la condición de fumador activo no jugaría un rol importante y que estaría asociado a factores que acompañan a estos pacientes, como la hipoxemia, las exacerbaciones y la masa grasa. Estos factores, potencialmente modificables, podrían eventualmente ayudar a reducir la inflamación. Sin embargo, las asociaciones observadas son débiles, indicando que la causa de la inflamación en la EPOC permanece largamente inexplicada y que la PCRus podría representar sólo uno de sus marcadores más que un factor activo en la progresión acelerada de la enfermedad y en su mortalidad.

\section{Referencias}

1. Rabe KF, Hurd S, Anzueto A, Barnes PJ, Buist SA, Calverley $\mathrm{P}$, et al. Global strategy for the diagnosis, management, and prevention of chronic obstructive pulmonary disease: GOLD executive summary. Am J Respir Crit Care Med 2007; 176: 532-55.

2. Chatila WM, Thomashow BM, Minai OA, Criner GJ, Make BJ. Comorbidities in Chronic Obstructive Pulmonary Disease. Proc Am Thorac Soc 2008; 5: 549-55.

3. Gan WQ, Man SFP, Senthilselvan A, Sin DD. Association between chronic obstructive pulmonary disease and systemic inflammation: a systematic review and a metaanalysis. Thorax 2004; 59: 574-80.

4. Rennard SI. Inflammation in COPD: a link to systemic comorbidities. Eur Respir Rev 2007; 16: 91-7.

5. Donaldson GC, Seemungal TAR, Patel IS, Bhowmik A, Wilkinson TMA, Hurst JR, et al. Airway and systemic inflammation and decline in lung function in patients with COPD. Chest 2005; 128: 1995-2004.

6. Dahl M, Vestbo J, Lange P, Bojesen SE, Tybjaerg-Hansen A, Nordestgaard BG. C-reactive protein as a predictor of prognosis in chronic obstructive pulmonary disease. Am J Respir Crit Care Med 2007; 175: 250-5.

7. Mannino DM, Thorn D, Swensen A, Holguin F. Prevalence and outcomes of diabetes, hypertension and cardiovascular disease in COPD. Eur Respir J 2008; 32: 962-9.

8. Rosenberg SR, Kalhan R. Dying from, and with, chronic obstructive pulmonary disease. Am J Respir Crit Care Med 2011; 183: 960-2.

9. Pepys MB, Hirschfield GM. C-reactive protein: a critical update. J Clin Invest 2003; 111: 1805-12.

10. Gan WQ, Man SFP, Sin DD. The interactions between cigarette smoking and reduced lung function on systemic inflammation. Chest 2005; 127: 558-64.

11. de Torres JP, Cordoba-Lanus E, López-Aguilar C, Muros de Fuentes M, Montejo de Garcini A, Aguirre-Jaime A, et al. C-reactive protein levels and clinically important predictive outcomes in stable COPD patients. Eur Respir J 2006; 27: 902-7.

12. Wouters EFM, Groenewegen KH, Dentener MA, Vernooy JHJ. Systemic inflammation in chronic obstructive pulmonary disease: The role of exacerbations. Proc Am Thorac Soc 2007; 4: 626-34.

13. Agusti AGN, Noguera A, Sauleda J, Sala E, Pons J, Busquets X. Systemic effects of chronic obstructive pulmonary disease. Eur Respir J 2003; 21: 347-60.

14. Papaioannou AI, Mazioti A, Kiropoulos T, Tsilioni I, Koutsokera A, Tanou K, et al. Systemic and airway inflammation and the presence of emphysema in patients with COPD. Respir Med 2010; 104: 275-82.

15. Eagan TML, Ueland T, Wagner PD, Hardie JA, Mollnes TE, Damas JK, et al. Systemic inflammatory markers in COPD: results from the Bergen COPD Cohort Study. Eur Respir J 2010; 35: 540-8.

16. Sin DD, Man SFP. Why are patients with chronic obstructive pulmonary disease at increased risk of cardiovascular diseases?: The potential role of systemic inflammation in chronic obstructive pulmonary disease. Circulation 2003; 107: 1514-9.

17. Schols AM, Buurman WA, Staal van den Brekel AJ, Dentener MA, Wouters EF. Evidence for a relation between metabolic derangements and increased levels of inflammatory mediators in a subgroup of patients with chronic obstructive pulmonary disease. Thorax 1996; 51: 819-24.

18. Poulain M, Doucet M, Drapeau V, Fournier G, Tremblay A, Poirier P, et al. Metabolic and inflammatory profile in obese patients with chronic obstructive pulmonary disease. Chron Respir Dis 2008; 5: 35-41.

19. Heikkilä K, Ebrahim S, Lawlor DA. A systematic review of the association between circulating concentrations of $\mathrm{C}$ reactive protein and cancer. J Epidemiol Community Health 2007; 61: 824-33.

20. Anisman H, Merali Z, Hayley S. Neurotransmitter, peptide and cytokine processes in relation to depressive disorder: Comorbidity between depression and neurodegenerative disorders. Prog Neurobiol 2008; 85: 1-74.

21. Rana JS, Mittleman MA, Sheikh J, Hu FB, Manson 
JE, Colditz GA, et al. Chronic obstructive pulmonary disease, asthma, and risk of type 2 diabetes in women. Diabetes Care 2004; 27: 2478-84.

22. Pinto-Plata VM, Mullerova H, Toso JF, Feudjo-Tepie $\mathrm{M}$, Soriano JB, Vessey RS, et al. C-reactive protein in patients with COPD, control smokers and non-smokers. Thorax 2006; 61: 23-8.

23. Yanbaeva DG, Dentener MA, Spruit MA, HouwingDuistermaat JJ, Kotz D, Passos VL, et al. IL6 and CRP haplotypes are associated with COPD risk and systemic inflammation: a case-control study. BMC medical genetics 2009; 10: 23.

24. Díaz O, Morales A, Osses R, Klaassen J, Lisboa C, Saldías F. Six-minute walk test and maximum exercise test in cycloergometer in chronic obstructive pulmonary disease. Are the physiological demands equivalent? Arch Bronconeumol 2010; 46: 294-301.

25. Mahler DA, Wells CK. Evaluation of clinical methods for rating dyspnea. Chest 1988; 93: 580-6.

26. Rodríguez-Roisin R. Toward a consensus definition for COPD exacerbations. Chest 2000; 117: 398S-401S.

27. Khot UN, Khot MB, Bajzer CT, Sapp SK, Ohman EM, Brener SJ, et al. Prevalence of conventional risk factors in patients with coronary heart disease. JAMA 2003; 290: 898-904.

28. Miller MR, Hankinson J, Brusasco V, Burgos F, Casaburi $\mathrm{R}$, Coates A, et al. Standardisation of spirometry. Eur Respir J 2005; 26: 319-38.

29. Hankinson J, Odencrantz J, Fedan K. Spirometric reference values from a sample of the general U.S. population. Am J Respir Crit Care Med 1999; 159: 179-87.

30. Lisboa C, Leiva A, Pinochet R, Repetto P, Borzone G, Díaz O. Valores de referencia de la capacidad inspiratoria en sujetos sanos no fumadores mayores de 50 años. Arch Bronconeumol 2007; 43: 485-9.

31. Lisboa C, Barría P, Yáñez J, Aguirre M, Díaz O. La prueba de caminata en seis minutos en la evaluación de la capacidad de ejercicio en pacientes con enfermedad pulmonar obstructiva crónica. Rev Med Chile 2008; 136: 1056-64.

32. Madani A, De Maertelaer V, Zanen J, Gevenois PA. Pulmonary emphysema: Radiation dose and section thickness at multidetector CT quantification Comparison with macroscopic and microscopic morphometry. Radiology 2007; 243: 250-7.

33. Bernard S, Leblanc P, Whittom F, Carrier G, Jobin J, Belleau R, et al. Peripheral muscle weakness in patients with chronic obstructive pulmonary disease. Am J Respir Crit Care Med 1998; 158: 629-34.

34. Morales A, Dreyse J, Díaz O, Saldías F, Carrasco M, Lisboa C. Systemic inflammation among stable ex smokers with chronic obstructive pulmonary disease. Rev Med Chile 2010; 138: 957-64.

35. Bazzano LA, He J, Muntner P, Vupputuri S, Whelton PK. Relationship between cigarette smoking and novel risk factors for cardiovascular disease in the United States. Ann Intern Med 2003; 138: 891-7.

36. Dahl M, Vestbo J, Zacho J, Lange P, Tybjaerg-Hansen A, Nordestgaard BG. C reactive protein and chronic obstructive pulmonary disease: a Mendelian randomisation approach. Thorax 2011; 66: 197-204.

37. van Durme YMTA, Verhamme KMC, Aarnoudse A-JLHJ, Van Pottelberge GR, Hofman A, Witteman JCM, et al. C-Reactive protein levels, haplotypes, and the risk of incident chronic obstructive pulmonary disease. Am J Respir Crit Care Med 2009; 179: 375-382.

38. Sabit R, Thomas P, Shale DJ, Collins P, Linnane SJ. The effects of hypoxia on markers of coagulation and systemic inflammation in patients with COPD. Chest 2010; 138: 47-51.

39. Takabatake $\mathrm{N}$, Nakamura $\mathrm{H}$, Abe $\mathrm{S}$, Inoue $\mathrm{S}$, Hino $\mathrm{T}$, Saito $\mathrm{H}$, et al. The relationship between chronic hypoxemia and activation of the tumor necrosis factor-alpha system in patients with chronic obstructive pulmonary disease. Am J Respir Crit Care Med 2000; 161: 1179-84.

40. Agusti A, Soriano JB. Dynamic hyperinflation and pulmonary inflammation: a potentially relevant relationship? Eur Respir Rev 2006; 15: 68-71.

41. Thorand B, Baumert J, Döring A, Herder C, Kolb H, Rathmann W, et al. Sex differences in the relation of body composition to markers of inflammation. Atherosclerosis 2006; 184: 216-24.

42. Eagan TML, Aukrust P, Ueland T, Hardie JA, Johannessen A, Mollnes TE, et al. Body composition and plasma levels of inflammatory biomarkers in COPD. Eur Respir J 2010; 36: 1027-33.

43. Liew SM, Doust J, Glasziou P. Cardiovascular risk scores do not account for the effect of treatment: a review. Heart 2011; 97: 689-97.

44. Brindle P, Beswick A, Fahey T, Ebrahim S. Accuracy and impact of risk assessment in the primary prevention of cardiovascular disease: a systematic review. Heart 2006; 92: 1752-9.

45. Kaptoge S, Di Angelantonio E, Lowe G, Pepys MB, Thompson SG, Collins R, et al. C-reactive protein concentration and risk of coronary heart disease, stroke, and mortality: an individual participant meta-analysis. Lancet 2010; 375: 132-40.

46. Zhang J, Rutten FH, Cramer MJ, Lammers JW, Zuithoff NP, Hoes AW. The importance of cardiovascular disease for mortality in patients with COPD: a prognostic cohort study. Family Practice 2011; 28: 474-81. 